\title{
Combining Cloud Computing and Artificial Intelligence Scene Recognition in Real-time Environment Image Planning Walkable Area
}

\author{
Jia-Shing Sheu*, Chen-Yin Han \\ Department of Computer Science, National Taipei University of Education, Taipei, Taiwan \\ Received 18 May 2019; received in revised form 11 June 2019; accepted 20 August 2019 \\ DOI: https://doi.org/10.46604/aiti.2020.4284
}

\begin{abstract}
This study developed scene recognition and cloud computing technology for real-time environmental imagebased regional planning using artificial intelligence. TensorFlow object detection functions were used for artificial intelligence technology. First, an image from the environment is transmitted to a cloud server for cloud computing, and all objects in the image are marked using a bounding box method. Obstacle detection is performed using object detection, and the associated technique algorithm is used to mark walkable areas and relative coordinates. The results of this study provide a machine vision application combined with cloud computing and artificial intelligence scene recognition that can be used to complete walking space activities planned by a cleaning robot or unmanned vehicle through real-time utilization of images from the environment.
\end{abstract}

Keywords: TensorFlow, computer vision, neural network, scene recognition, cloud computing

\section{Introduction}

With the rapid development of technology, high-quality digital cameras have become commonly used for acquiring images. These cameras are used in mobile phones, personal digital assistants, robots, medical systems, and surveillance and home security systems. With machine learning and neural operations, the image in the cloud can be easily identified. This study primarily used cloud resources currently available to upload real-time images from the environment obtained using a digital camera to the cloud and cooperate with cloud machine learning and image recognition for identifying different objects in the real-time image. When objects in a live image can be successfully identified, the environmental conditions observed in the live image can be further distinguished. This study used these resources to map real-time images from the environment to walkable areas by combining images obtained using digital cameras, which can be used with eye-like cameras when combined with cloud machine learning and neural recognition.

In the study, Section 2 provides a review of the literature and methodology. The structure of the system is discussed in Section 3. Design and experiments are provided in Section 4. Finally, conclusions are provided in Section 5.

\section{Literature Review and Methodology}

An artificial neural network is a mathematical model based on a neural network system that mimics humans. The neural network aims to establish a neural network model that mimics biological perception in a computer and uses this mathematical statistical model to optimize data learning. In the cognitive system of artificial intelligence, the use of neural network-like algorithms is more advantageous than formal logic inference algorithms. Because the neural network has nonfixed, nonconvex, nonlinear, and nonlimiting characteristics, different structures, functions, and learning algorithms can be used [1-3]. Neural networks can be classified into various types, such as perceptual neural networks, linear neural

* Corresponding author. E-mail address: jiashing@tea.ntue.edu.tw

Tel.: +886-2-27321104 ext. 55425; Fax: +886-2-27375457 
networks, inverted-transitive neural networks, and enhanced neural networks. Because an inverse-transfer-like neural network can approximate any function with arbitrary precision under appropriate parameter settings, it can effectively predict the problem and provide functions such as environment perception, pattern recognition, and prediction. Neural networks have been applied in many fields, including emotion perception, handwriting recognition, image recognition, and language translation [4-7].

A neural network generally has several levels, and each level can provide a sufficient number of neurons. Each neuron adds up the input of the previous layer of neurons and enters the conversion of an activation function. Each neuron has a distinct connection with the neurons of the subsequent layer, and thus, the output value of the upper layer of neurons is weighted and passed to the neurons of the subsequent layer. The neurons in the same layer are not connected and are distinct [8-9].

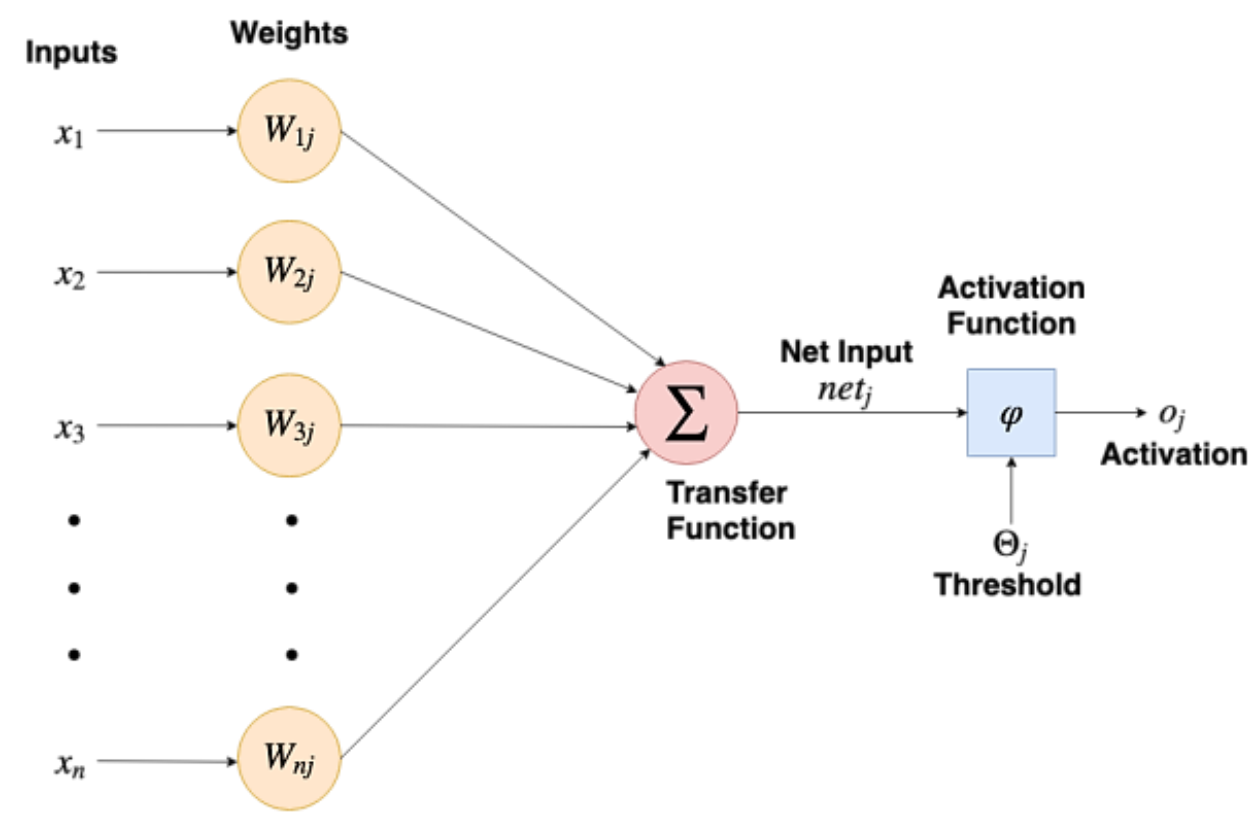

Fig. 1 Neural network-like simulation of how a single neuron operates

The neural network is represented by numerous single neurons as shown in Fig. 1, and comprises the following components:

(1) Inputs: Receive signals from external or upper neurons.

(2) Weights: The memory of the neural network. The number of weights corresponds to the number of inputs in the input layer; that is, one weight corresponds to one input, which is multiplied by a conversion function and then added to the other values.

(3) Transfer function: The transfer function accumulates the product of all input values and weight values and then enters the activation function.

(4) Threshold: Each neuron has a threshold. When the product value is accumulated by the conversion function, the offset value is added. The excitation function is provided to determine whether the signal needs to be output downward. That is, partial migration is used to control the continuation of the value.

(5) Activation function: An excitation function of the neural network introduces the characteristics of the nonlinear output, and thus, the neural network can perform mapping to learn complex functions

(6) Outputs/Activation: The output values mapped using the excitation function can be used as inputs to the subsequent layer of neurons. 
The architecture of the neural network connects the aforementioned single neurons to form a model. Some commonly used neural network architectures are inverted neural networks, convolutional neural networks, and recurrent neural networks.

\section{System Structure}

The study is primarily based on a neural network-based regional identification system, its focus being on detecting walkable areas. By inputting images to a pre-trained neural network, the objects in the images can be identified. The empty areas between objects indicate walkable areas. Fig. 2 presents the architecture of the system obtained from using IEF0 [10]. To achieve to mark walkable areas and relative coordinates, this study is divided into three parts:

(1) The first part constitutes neural network object identification. When the image enters the neural network, the trained model is used to load the images into the personal computer, identify the object position, and predict the score and object name.

(2) The second part involves finding the path. By using the object position and coordinates obtained using the neural network, the distance between the objects is calculated, and the shortest distance between objects is identified to determine the position of the empty area as shown in Fig. 3.

(3) The third part includes path marking. The two points with the shortest path are connected. The enclosed space inside the line is the walkable area primarily discussed in this paper as shown in Fig. 4.

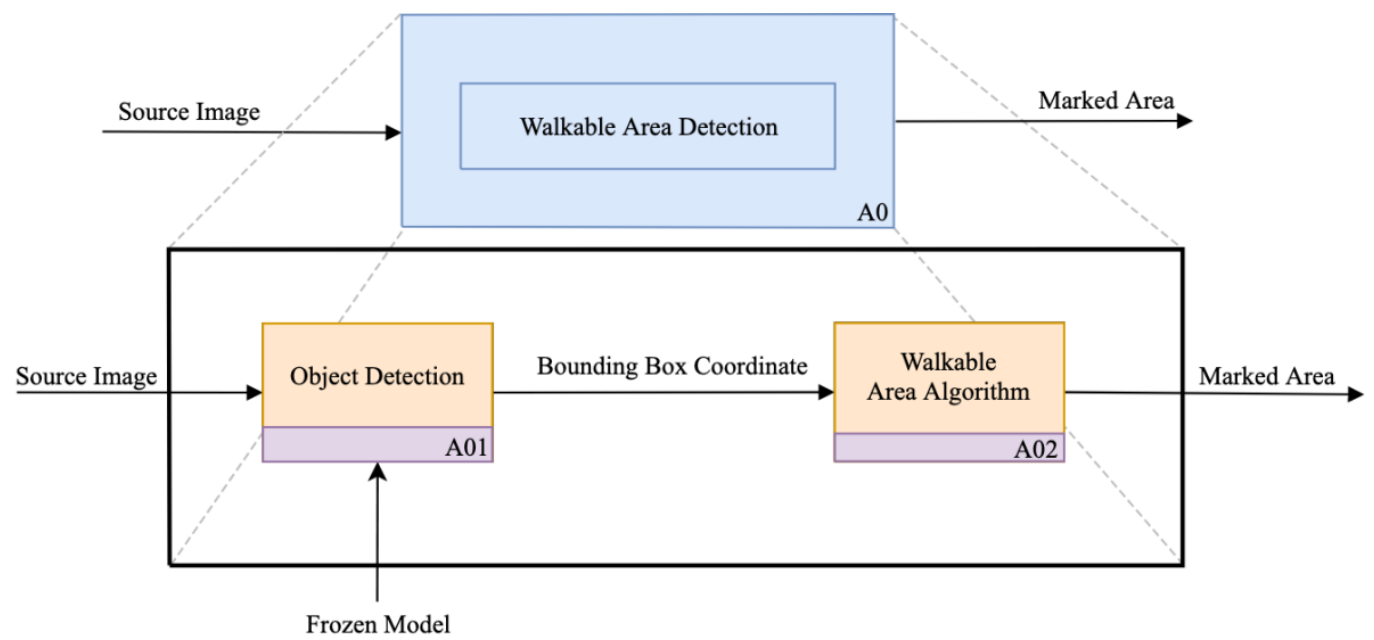

Fig. 2 The architecture of the whole system

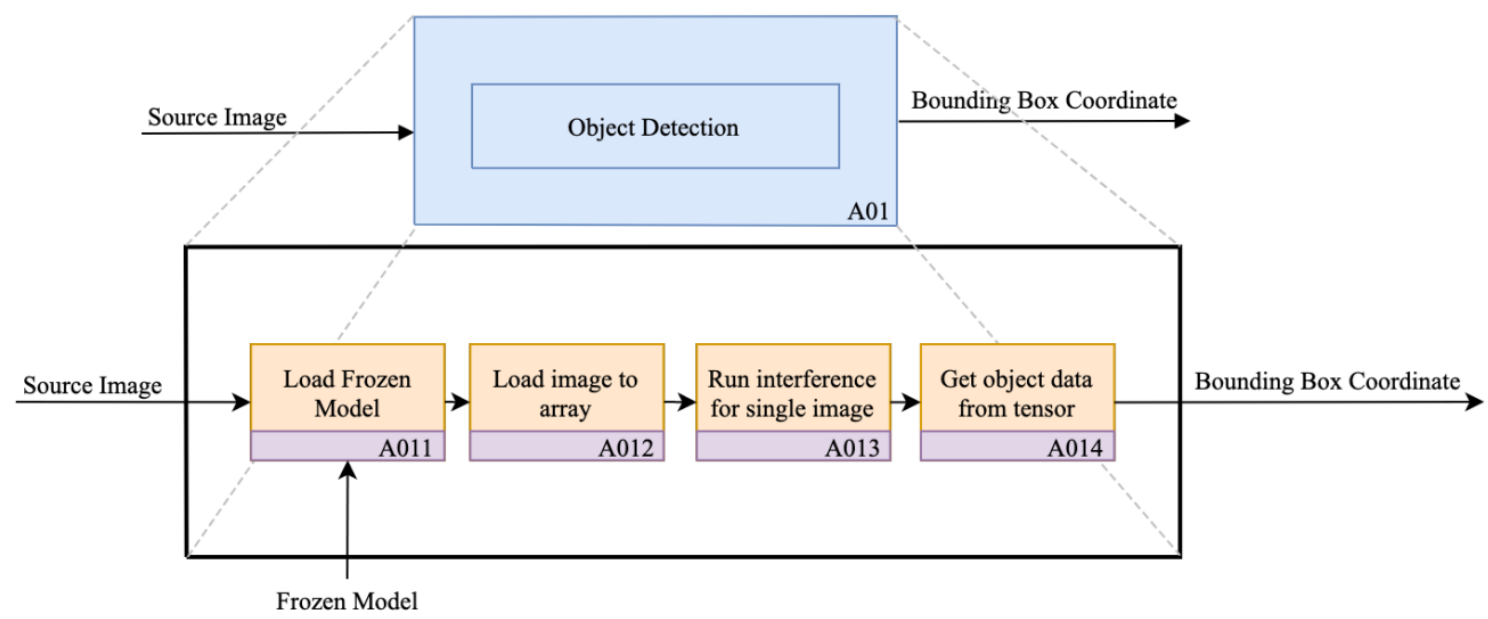

Fig. 3 The architecture of object detection 


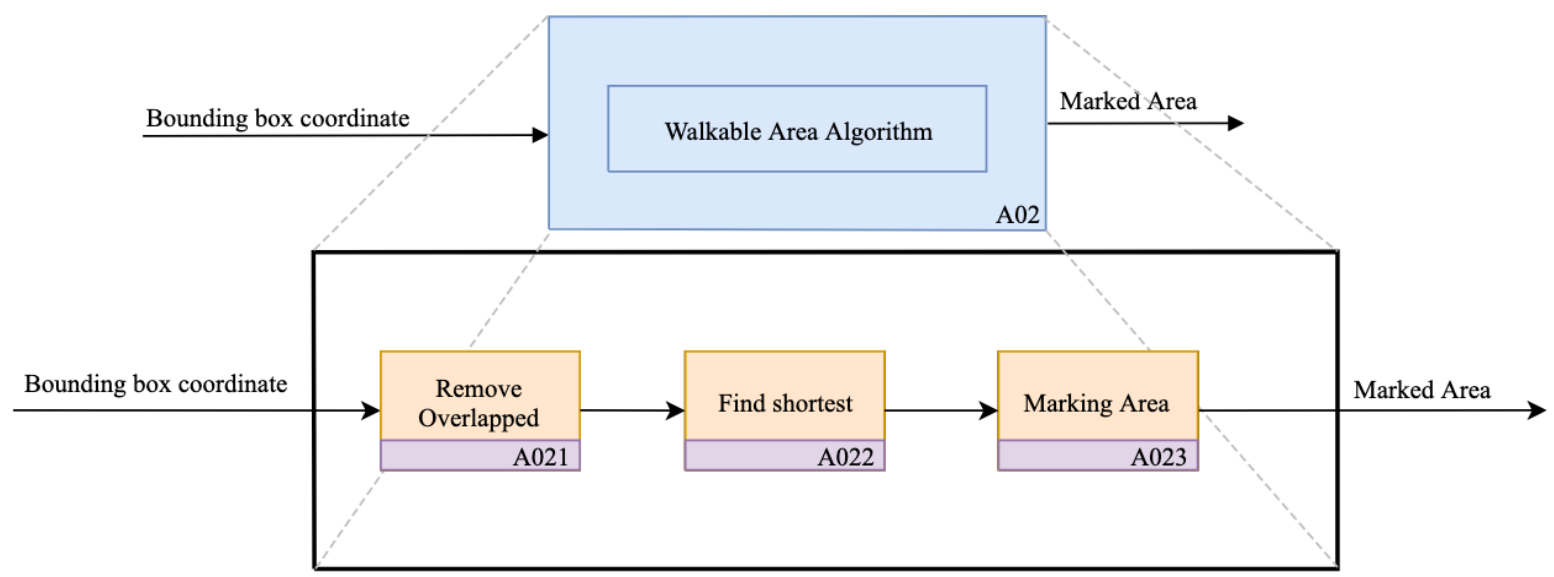

Fig. 4 The architecture of walkable area marked

This experiment used TensorFlow as the core machine learning framework and an open source project TensorFlow object detection application programming interface [11] as the core of object recognition, where the source code allows the use of trained models that meet their requirements.

The pretraining model compiled using the TensorFlow detection model zoo was used in this study. The models used for object recognition were faster_rcnn_inception_v2_atrous_coco, ssd_mobilenet_v1_coco, and ssd_mobilenet_v1_fpn_coco.

With the bounding box coordinates of the object, the distance between all bounding boxes can be calculated. The algorithm uses the distance between each point to select the shortest path, which is considered a part of the walkable area and is connected into a large block through the plurality of lines. According to an axiom in the Euclidean geometry system, "there are two points and only one line," and each bounding box has four points. Thus, for $\mathrm{n}$ bounding boxes, the following general formula can be used in Eq. (1):

$$
\left(\begin{array}{l}
4 n \\
2
\end{array}\right)-n \times 2-n \times 4
$$

In any two bounding boxes, distance D between any two points is measured, which can be recorded as $\boldsymbol{P}_{\mathbf{1}}\left(\boldsymbol{x}_{\mathbf{1}}, \boldsymbol{y}_{\mathbf{1}}\right)$ and $\boldsymbol{P}_{\mathbf{2}}\left(\boldsymbol{x}_{2}, \boldsymbol{y}_{2}\right)$. The distance between all points is calculated using Eq. (2). For each length comparison, the two shortest distance between all bounding boxes is selected, and starting and ending point coordinates are recorded.

$$
D=\sqrt{\left(x_{2}-x_{1}\right)^{2}-\left(y_{2}-y_{1}\right)^{2}}
$$

The start and end point coordinates are obtained using the aforementioned method, and the line connecting the points can form a closed area, which is the walkable area discussed by the research institute.

\section{Experimental Results}

Table 1 Graph representations

\begin{tabular}{|c|c|}
\hline Components & Specification \\
\hline Operating System & Debian 9 \\
\hline Central Processing Unit & AMD Ryzen 7 2700x \\
\hline Random Access Memory & DDR4-3200 16GB \\
\hline Graphics Processing Unit & NVIDIA GTX1080Ti \\
\hline
\end{tabular}

A car combined the Raspberry Pi and CMOS camera is used to capture the environmental image as shown in Fig. 5. The Raspberry $\mathrm{Pi}$ is a popular embedded system, which is developed by the Raspberry Pi Foundation in the United Kingdom. The processor is Boardcom BCM2837 SoC with a 1.2GHz 64-bit quad-core ARM Cortex-A53 processor. Raspberry Pi provides 40-pin General Purpose Input-Output (GPIO) connector, that allows user connect peripherals such as LCD1602 
module, sensors, etc. via Inter-Integrated Circuit $\left(\mathrm{I}^{2} \mathrm{C}\right)$, Universal Asynchronous Receiver-Transmitter (UART) and Serial Peripheral Interface (SPI). Raspberry Pi also provides the accessories, such as touch panel and camera, the camera which is this experiment used, the camera can get $1024 \times 768$ images by 5 million pixels, it connects Raspberry Pi by Camera Serial Interface (CSI) with a flexible flat cable. Raspberry Pi's power rating is $5 \mathrm{~V} / 1.34 \mathrm{~A}(1.5 \mathrm{~W})$, we use a mobile power as a power source by MicroUSB, providing maximum 5V/2.4A (12W) output.

In this experiment, we use Wireless Local Area Network (WLAN) and Local Area Network (LAN) connect Raspberry Pi and server. Raspbian was chosen for the operating system, it is a Debian-based for Raspberry Pi, officially provided by Raspberry Pi Foundation. Raspbian is highly optimized for the Raspberry Pi line's low-performance ARM CPUs, also provide a desktop environment for beginners.

In the beginning, Raspberry Pi boots into the system, then startup streaming service, the protocol is using Real Time Streaming Protocol (RTSP). At the server side, the server captures the image from Raspberry Pi, TensorFlow object detection program will detect the object from the image, in this experiment, we use "faster_rcnn_inception_ resnet_v2_atrous_coco" as frozen model to detect object, the reason we chose this model, it can recognize object more than the other two frozen model, but disadvantage of this model is using more time. After detected, the program will generate object coordinate, name and detected score, to validate our theory, we need coordinate data to calculate the walkable area, then, the program will send object coordinate data and source imaged to the walkable area program, name and score data are optional data, but in this experiment, we didn't use them, those data contain the object name and detected score.

The walkable area program was used in Python 3 as programming language, in this program, coordinate data from TensorFlow object detection is playing an important part in this experiment, first step, we use the coordinate data to mark the bounding box, second step, using the algorithm to find the walkable area of this image, then, the program will generate the marked image.

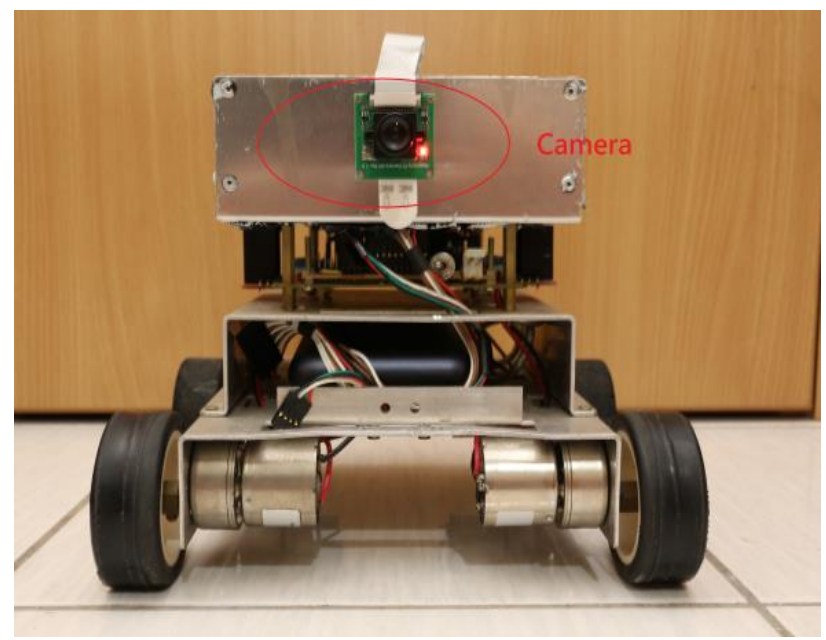

(a) The Raspberry Pi

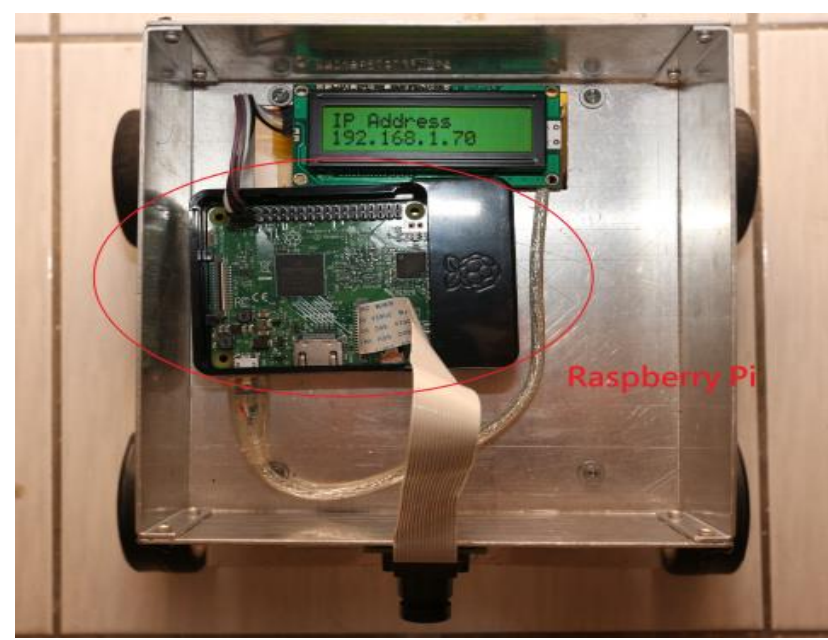

(b) CMOS camera

Fig. 5 A car combined the Raspberry Pi and CMOS camera

Figs. (6-7) present the original input, result detected using TensorFlow object detection, and walkable area determined using the program, respectively. The program was developed according to the aforementioned theory. Fig. 7 shows the result of the system output, bounding box in green represent the object, the two red lines represent the two shortest distance between two bounding boxes, two lines means we have four coordinates. To show the walkable area, creating an empty layer, same width and height as origin image, using these coordinates to create a polygon on it, filling in blue color in a closed area, after creating the layer, it will overlay on the origin image. The marked area is the walkable area that the system determined. 


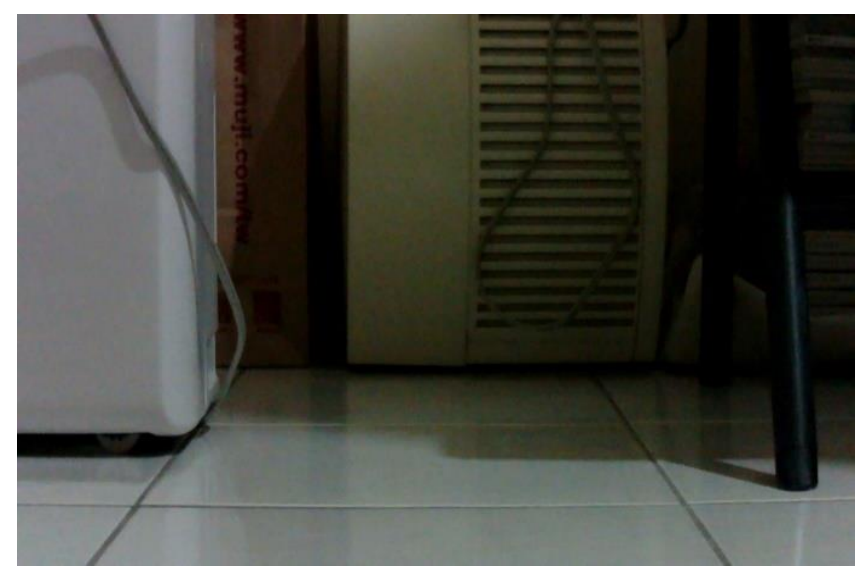

Fig. 6 The environmental image captured by the CMOS camera

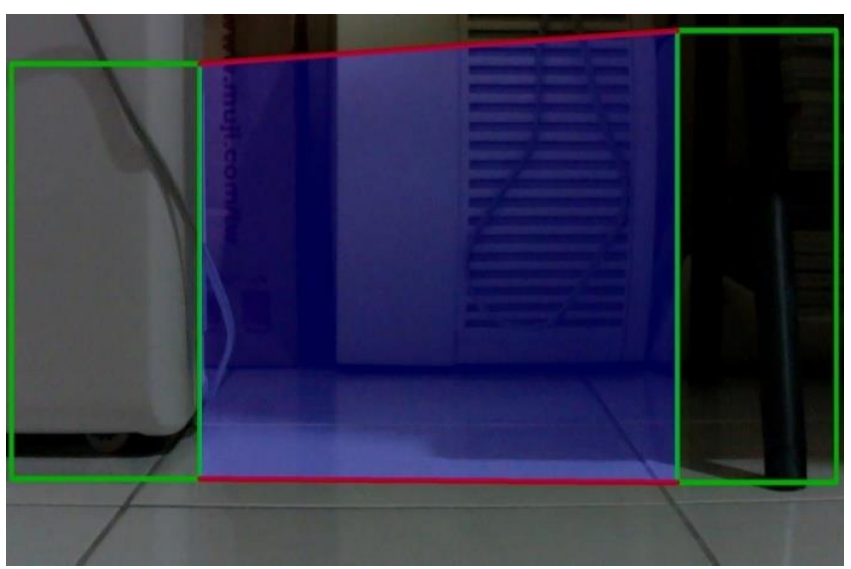

Fig. 7 The walkable area determined using the system marked by BLUE COLOR

In Fig. 8, this environment is a walking area in the laboratory, in human thinking, we can pass through by the box's lefthand side, but there was a wheel of the chair in the left, so we pass through between these two objects. The program will detect the objects using Fig. 8, then the program used an algorithm to calculate the walkable area between these two objects, shown in Fig. 9. You can see that the program marks the walkable area by blue color, so does human thinking. But in Fig. 9, some object is not marked, for example, the cabinet in the middle of Fig. 9, algorithm skip that object, the blue area is overlapped on it.

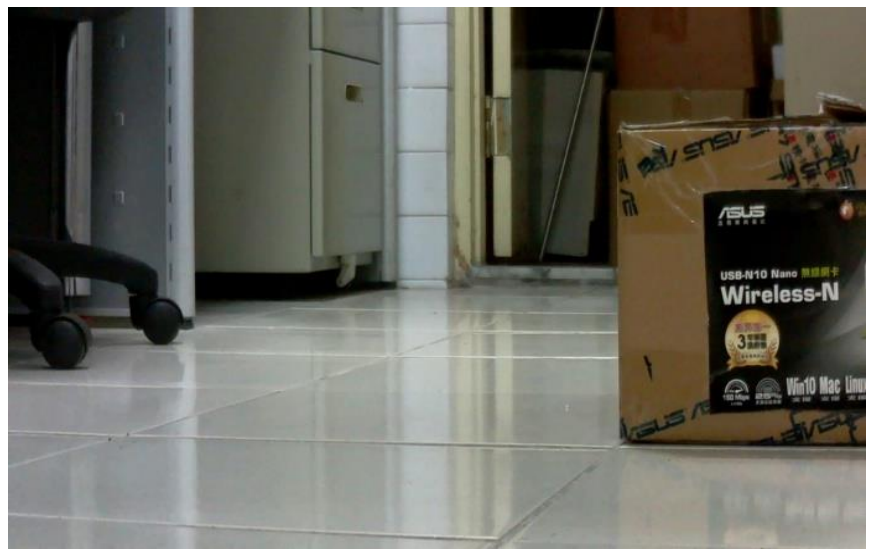

Fig. 8 The environmental image captured by the CMOS camera

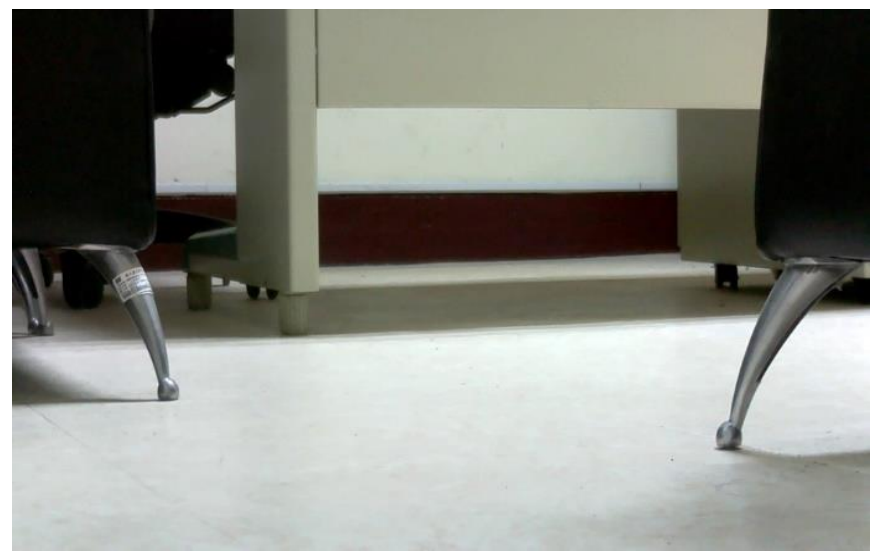

Fig. 10 The environmental image captured by the CMOS camera

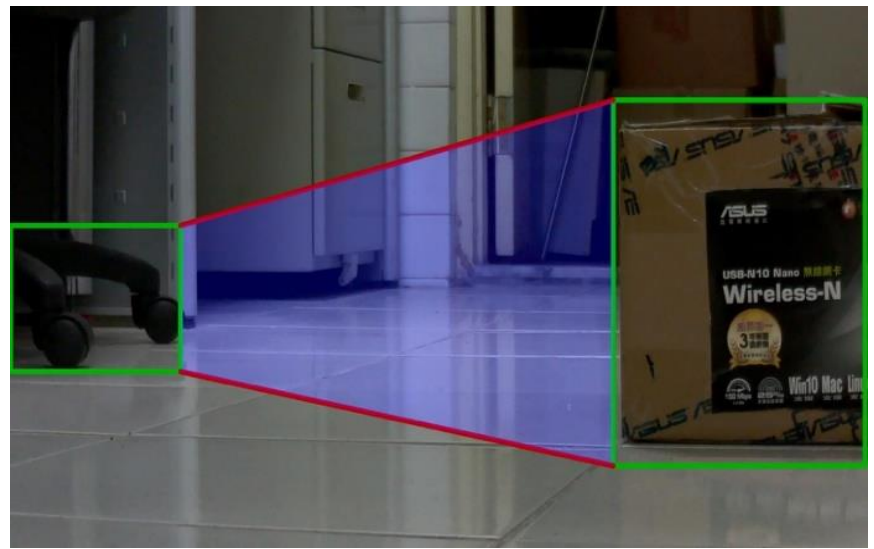

Fig. 9 The walkable area determined using the system, marked by BLUE COLOR

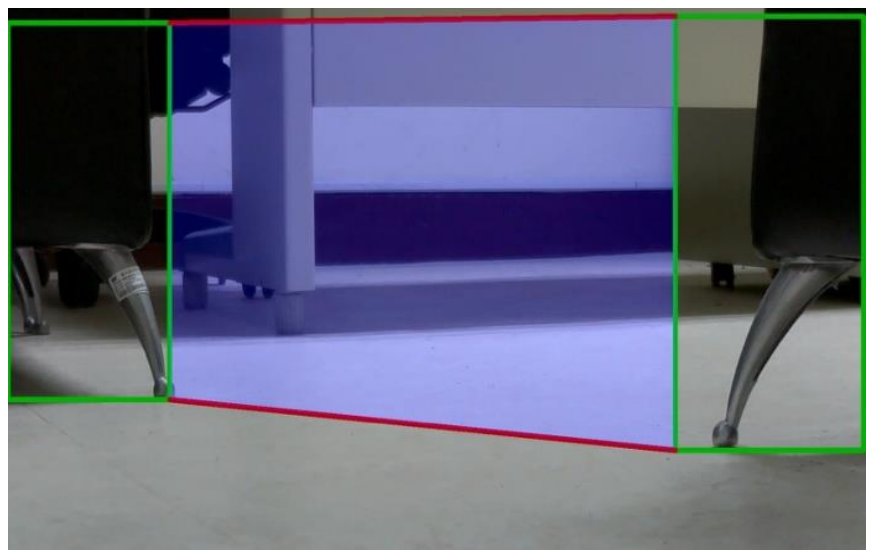

Fig. 11 The walkable area determined using the system, marked by BLUE COLOR

Table 2 presents the detail of the detection, we chosen Google's Tensorflow Object detection API "faster_rcnn_ inception_resnet_v2_atrous_coco" frozen model as object detection model, "ssd_mobilenet_v1_coco," and "ssd_mobilenet 
_v1_ fpn_coco" are not used here, two of them can't recognize more than two objects, by this reason, the algorithm needs two or more objects input, which is the area that we want to find.

Table 2 Detection details for Figs. $(7,9,11)$

\begin{tabular}{|c|c|l|c|}
\hline & Number of detections & \multicolumn{1}{|c|}{ Detection classes } & Time (sec.) \\
\hline Fig. 7 & 4 & Toilet, microwave, oven, chair & 0.0157 \\
\hline Fig. 9 & 4 & Refrigerator, chair, bowl & 0.0161 \\
\hline Fig. 11 & 2 & Chair & 0.0191 \\
\hline
\end{tabular}

By experiment results, this algorithm needs more object data, accuracy will be increased. Choosing a model is a subject in this study, we can train the model base on the existing model, so we should prepare the object that appears indoor frequently, such as sofa, chairs, cabinet, fans, computers, etc. Another problem is, vision is limited, the camera cannot capture whole object, only a part of an object, chair foot is an obvious case, sometimes object detection cannot detect what the object is, or it is an object, cause the program cannot find the walkable area. The problem as descript above mentioned, this problem is future work that we need to solve them.

\section{Conclusions}

This study proposed the use of recent paths between all objects to determine the walkable area, the experiment showed the result, it proofs the algorithm can find the walkable area by TensorFlow object detection. Enhanced this algorithm is the most important job in the future, low latency is the goal of this algorithm, the real-time system does not allow high latency. For example, the cleaning robot needs to know which area cannot entry, low latency can speed up the efficiency of the cleaning work.

With the popularity of high-bandwidth networks, cloud computing was used to obtain the desired results. With technological developments, the tensor calculation of embedded systems can considerably improve computing efficiency. In the near future, this algorithm can be installed in embedded systems, which will be a significant development for robot vision.

\section{Conflicts of Interest}

The authors declare no conflict of interest.

\section{References}

[1] S. Ren, K. He, R. Girshick, and J. Sun, "Faster R-CNN: towards real-time object detection with region proposal networks," IEEE Transactions on Pattern Analysis and Machine Intelligence, vol. 39, no. 6, pp. 1137-1149, 2017.

[2] T. Y. Lin, M. Maire, S. Belongie, L. Bourdev, R. Girshick, J. Hays, P. Perona, D. Ramanan, C. L. Zitnick, and P. Dollár, "Microsoft COCO: common objects in context," arXiv:1405.0312v3 [cs. CV], February 2015.

[3] A. G. Howard, M. Zhu, B. Chen, D. Kalenichenko, W. Wang, T. Weyand, M. Andreetto, and H. Adam, "MobileNets: efficient convolutional neural networks for mobile vision applications," arXiv:1704.04861 [cs. CV], 2017.

[4] R. Girshick, J. Donahue, T. Darrell, and J. Malik, "Rich feature hierarchies for accurate object detection and semantic segmentation," Proc. The IEEE Conference on Computer Vision and Pattern Recognition (CVPR), IEEE Press, June 2014, pp. 580-587.

[5] W. Liu, D. Anguelov, D. Erhan, C. Szegedy, S. Reed, C. Y. Fu, and A. C. Berg, "SSD: single shot multibox detector," Proc. Part I: ECCV 2016-the 14th European Conference, Amsterdam, Netherlands, October 2016, pp. 21-37.

[6] K. He, X. Zhang, S. Ren, and J. Sun, “Deep residual learning for image recognition,” Proc. 2016 IEEE Conference on Computer Vision and Pattern Recognition (CVPR), IEEE Press, Jun. 2016, pp. 770-778.

[7] C. Ning, H. Zhou, Y. Song, and J. Tang, "Inception single shot multibox detector for object detection,” Proc. 2017 IEEE International Conference on Multimedia \& Expo Workshops (ICMEW), IEEE Press, 2017, pp. 549-554.

[8] J. S. Sheu and Y. L. Huang, "Implementation of an interactive TV interface via gesture and handwritten numeral recognition," Multimedia Tools and Applications, vol. 75, no. 16, pp. 9685-9706, 2016. 
[9] R. Kaluri and P. Reddy, "Optimized feature extraction for precise sign gesture recognition using self-improved genetic algorithm,” International Journal of Engineering and Technology Innovation, vol. 8, no. 1, pp. 25-37, 2018.

[10] C. H. Chen, C. M. Kuo, C. Y. Chen, and J. H. Dai, "RETRACTED: the design and synthesis using hierarchical robotic discrete-event modeling," Journal of Vibration and Control, vol. 19, no. 11, pp. 1603-1613, 2012.

[11] M. Abadi, P. Barham, J. Chen, Z. Chen, A. Davis, J. Dean, M. Devin, S. Ghemawat, G. Irving, M. Isard, M. Kudlur, J. Levenberg, R. Monga, S. Moore, D. G. Murray, B. Steiner, P. Tucker, V. Vasudevan, P. Warden, M. Wicke, Y. Yu, and X. Zheng, “TensorFlow: a system for large-scale machine learning,” arXiv:1605.08695 [cs.CV], 2016.

Copyright $@$ by the authors. Licensee TAETI, Taiwan. This article is an open access article distributed under the terms and conditions of the Creative Commons Attribution (CC BY-NC) license (https://creativecommons.org/licenses/by-nc/4.0/). 